\title{
Editorial
}

\section{DISTINGUISHING BETWEEN RELAPSE AND LATE REVERSAL REACTION IN MULTIDRUG (MDT)-TREATED BT LEPROSY}

This issue contains a report by Shetty et al. on the incidence of viable Mycobacterium leprae in lesions presenting with reversal reaction. Dr Michael Waters comments on some of the issues raised.

The two great advances, which both occurred around 1960, and which presaged the dramatic increase in the understanding of leprosy and its treatment in the following 2 decades, were the development by Ridley and Jopling of their classification of leprosy according to immunity, ${ }^{1,2}$ and the discovery by Shepard of limited multiplication of $M$. leprae in the mouse foot pad. ${ }^{3}$ The latter was rapidly confirmed and energetically applied by Rees, ${ }^{4}$ so that the first cases of proven dapsone resistance were reported by $1964 .^{5}$

In those early days, and routinely still today, the Shepard school inoculated 5000 and the Rees school 10,000 leprosy bacilli into the mouse foot pad, but both reported harvests averaging around 1,000,000 bacilli after 6-8 months, with no subsequent increase in numbers once this plateau count had been reached. With further experience, it was realized that a minority of strains of $M$. leprae isolated from patients gave slightly higher yields of 5000 $10,000,000$, and another minority gave low yields of around 1-500,000 bacilli, and that these characteristics were maintained on passage (subculture) in normal mice. Rees and his colleagues $^{6}$ also found that, in immunodeficient mice, multiplication continued after 6 months, sometimes the inoculated footpad became swollen, and yields of up to $1,000,000,000\left(10^{9}\right)$ bacilli could be obtained; systemic spread of leprosy bacilli also occurred. For these early experiments, biopsies were taken from untreated or relapsed lepromatous (LL and BL) patients as the source of bacilli. I remember that workers at the Leprosy Research Unit, Sungei Buloh, Malaysia sent a few biopsies from smear-positive borderline-tuberculoid (BT) patients, but the tissue yields of $M$. leprae were usually very low, or even negative by normal counting methods, and the mouse foot pads at harvest were often negative, so that no more fresh BT tissues were sent at that time for mouse inoculation. I do not recall that any serious study by mouse footpad inoculation of strains of $M$. le prae isolated from BT patients was subsequently undertaken, although individual BT patients were biopsied for mouse studies for a variety of reasons.

As experience with the mouse footpad model developed, however, experiments were set up involving the inoculation of very tiny numbers of viable bacilli, especially in three areas. First, both Shepard ${ }^{7}$ and $\operatorname{Rees}^{8}$ did so, even though they still gave standard-sized inocula, 
when they obtained serial biopsies from patients commencing chemotherapy, for after the start of treatment the proportion of viable drops, whether dramatically with rifampicin or more slowly with dapsone and clofazimine. Second, tiny total numbers were injected in Colston's elegant Proportional Bactericidal Test. ${ }^{9}$ It was found, quite logically, that when very few viable bacilli were present in the inoculum, 'takes' (that is, proven multiplication of M. leprae at harvest) were patchy, involving only a few or only one foot pad(s) in a single group of mice, and that such multiplication was frequently not detected until 12 months after inoculation, due to the extra number of generations required for the bacilli to reach detectable and plateau numbers; therefore protocols required most groups of mice to be kept for 12 months. Third, in their work on microbial persistence, Rees and his colleagues inoculated suspensions from a variety of tissues taken from treated LL and BL patients into immunodeficient mice. Many of these suspensions were either 'negative' (that is, bacilli, if present, were below the limit of detection), or gave very low counts. Nevertheless, in our first study, made on 12 patients who had received 10-12 years treatment with dapsone monotherapy, we were able to isolate three strains which passaged to normal mice, and were shown to be fully sensitive to dapsone. ${ }^{10}$ However, in other experiments, we obtained a number of patchy counts of around 100,000 bacilli at 10-12 months in immunodeficient mice, and some of these strains failed to passage to normal mice; the explanation is by no means obvious. In all these experiments, Rees meticulously cultured the suspensions, usually on LowensteinJensen slopes, to rule out the possibility of contamination with saprophytic mycobacteria, as, for example, there is one species that can live in distilled water.

With this background in mind, let us now consider the important report by Shetty, Wakade and Antia in this issue. The authors remind us of the well-known difficulty of distinguishing between relapse and late reversal reaction in multidrug (MDT)-treated BT leprosy. The World Health Organization has suggested a trial course of corticosteroids in such 'relapses', on the assumption that therapeutic steroids will suppress a reversal reaction and prevent or ameliorate any associated nerve damage, whereas the treatment would only partially and temporarily suppress a reaction due to viable bacteria (and the latter would continue to multiply during this period of steroid immunosuppression, so that acid fast bacteria might be more easily detected in smears, though hopefully any further clinical spread would be detected early). One assumes, for the authors do not give their precise protocol, that they were hoping to be able to distinguish between the two conditions by the detection in relapses of viable leprosy bacilli by mouse footpad inoculation, not present in reversal reactions due to an immunological response to antigen(s) from dead bacilli, (though it must be remembered that senior clinicians had seen bacterial relapse in dapsone and thiambutosinetreated BT-BL patients first present as reversal reaction), and to ascertain if steroids were of value in the differential diagnosis.

From a careful examination of their data, there is clear prima facie evidence of multiplication of leprosy bacilli in five experiments, namely those involving patients 1,5 , 10,14 and 25. The results for patient 22 are less certain; although five of six foot pads gave counts of acid fast bacilli, the highest yield was only 140,000; was this a very low yielding strain, or were these counts analogous to the low counts obtained in persister studies, in which the strains of $M$. leprae did not passage? The patchy occasional counts obtained in some of the other experiments are of even more doubtful significance, especially those early positive counts at 6 or 8 months.

Of the five undoubted takes, four (1, 5, 10 and 25) had follow-up durations from release from MDT treatment (RFT) to relapse of 7, 9, 10 and 13 years, and from start of MDT to 
relapse of 10,11, 12 and 15 years. Only one patient, no. 14, who had received PB-MDT for 6 months, had had a short follow-up duration to relapse of 2.5 years. Three of the first four had received PB-MDT; only one (no. 25) had received MB-MDT, and he had had the longest duration from RFT to relapse of 13 years.

Surely, these data are not surprising, when one considers the size of the leprosy problem 20 years ago in the Mumbai (Bombay) region, though it would be helpful if the authors could give some estimate of the size of the tuberculoid or BT pool from which their special patients were obtained. Furthermore, it is known that relapses in BT leprosy can occur very late. I have myself briefly reported a patient who had a single, large anaesthetic lesion on his thigh at age 36, treated then so successfully with injections of hydnocarpus oil that he was not given dapsone when the drug became available, and who relapsed 40 years later, at the age of 76 , with a new tuberculoid lesion on his face. ${ }^{11}$

In retrospect, it would have been very interesting to extend the authors' protocol, to include the passage of these five or six strains of M. leprae into fresh mice, both to confirm their viability, and more especially to study their drug sensitivities. For example, was strain no. 14 dapsone resistant, so that, in effect, he had received monotherapy with six doses of rifampicin? Were any of the five or six strains rifampicin resistant: Grosset and his colleagues have suggested that to select rifampicin resistant mutants, more than six, and possibly as many as 50 doses of rifampicin need to be administered; ${ }^{12}$ all the authors' other positive strains had been subjected to at least 18 months of MDT, although one might hope that no rifampicin resistant mutants were present in the comparatively tiny bacterial load present in many BT patients. Furthermore, if any strain had been found to be rifampicin resistant, it would have been helpful to have the finding confirmed, either in another mouse footpad laboratory or by PCR, such collaborative tests being arranged, if necessary, through the World Health Organization. Outside confirmation is always helpful in chemotherapy, as the tragedy of the two reports of clofazimine resistance demonstrates; ${ }^{13,14}$ one strain was never sent elsewhere, and the other was held up in transit. Such collaborative planning is best built into the original protocol, and if the authors extend their important studies, as many will hope, perhaps these suggestions may be included.

But already the authors have produced good evidence that viable leprosy bacilli can cause late relapse in BT leprosy, and that such relapses may be associated with reversal reactions. We shall welcome their further studies.

\section{References}

${ }^{1}$ Ridley DS, Jopling WH. A classification of leprosy for research purposes. Lepr Rev, 1962; 33: 119-128.

2 Ridley DS, Jopling WH. Classification of leprosy according to immunity: a five group system. Int J Lepr, 1966; 34: $255-273$.

3 Shepard CC. The experimental disease that follows the injection of human leprosy bacilli into foot pads of mice. $J$ Exp Med, 1960; 112: 445-454.

${ }^{4}$ Rees RJW. Limited multiplication of acid-fast bacilli in the foot pads of mice inoculated with Mycobacterium leprae. Br J Exp Pathol, 1964; 45: 207-214.

5 Pettit JHS, Rees RJW. Sulphone resistance in leprosy. An experimental and clinical study. Lancet, 1964; ii: 673674.

${ }^{6}$ Rees RJW, Waters MFR, Weddell AGM, Palmer E. Experimental lepromatous leprosy. Nature, 1967; 215: 599602.

7 Shepard CC. A brief review of experiences with short-term clinical trials monitored by mouse foot-pad inoculation. Lepr Rev, 1981; 52: 299-308.

8 Rees RJW, Pearson JMH, Waters MFR. Experimental and clinical studies on rifampicin in reatment of leprosy. Br Med J, 1970; 1: 89-92. 
${ }^{9}$ Colston MJ, Hilson GRF, Banerjee DK. The 'Proportional Bactericidal Test': a method for assessing bactericidal activity of drugs against Mycobacterium leprae in mice. Lepr Rev, 1978; 49: 7-15.

10 Waters MFR, Rees RJW, McDougall AC, Weddell AGM. Ten years of dapsone in lepromatous leprosy: clinical, bacteriological and histological assessment and the finding of viable leprosy bacilli. Lepr Rev, 1974; 45: $288-298$.

11 Waters MFR. The chemotherapy of leprosy. In: Ratledge C, Stannford JL, Grange J (eds) The biology of the mycobacteria, volume 3. Academic Press, London, 1989, pp 405-474.

12 Grosset JH, Guelpa-Lauras C-C, Bobin P, Brucker G et al. Study of 39 documented relapses of multibacillary leprosy after treatment with rifampicin. Int J Lepr, 1989; 57: 607-614.

13 Kar HK, Bhatia VN, Harikrishnan S. Combined clofazimine- and dapsone resistant leprosy. A case report. Int J Lepr, 1986; 54: 389-391.

14 Levy L. Clofazimine resistant M. leprae (Letter). Int J Lepr, 1986; 54: 137-140.

3 Regents Close, Radlett, Herts WD7 7DB, UK

M. F. R. WATERS 